\title{
Herramientas de visualización gráfica online para datos abiertos presupuestarios de administraciones públicas
}

\author{
Jesús García-García*, María Isabel Alonso-Magdaleno** \\ *Departamento de Contabilidad. Universidad de Oviedo \\ Facultad de Economía y Empresa. \\ Correo-e: jesgar@uniovi.es ORCID iD: http://orcid.org/0000-0002-5120-8851 \\ **Departamento de Administración de Empresas. Universidad de Oviedo. \\ Facultad de Economía y Empresa. \\ Correo-e: ialonso@uniovi.es ORCID iD: http://orcid.org/0000-0002-4865-1561
}

Recibido: 23-05-20; 2a versión: 17-06-20; Aceptado: 19-06-20; Publicado: 04-06-2021

Cómo citar este artículo/Citation: García-García, J.; Alonso-Magdaleno, M. I. (2021). Herramientas de visualización gráfica online para datos abiertos presupuestarios de administraciones públicas. Revista Española de Documentación Científica, 44 (3), e305. https://doi.org/10.3989/redc.2021.3.1792

Resumen: La divulgación de información pública por parte de los gobiernos y administraciones resulta de manera general excesivamente técnica quedando fuera del alcance y comprensión de la mayor parte de la ciudadanía ante la que deben rendir cuentas e integrar en sus procesos participativos. Los avances en las políticas de datos abiertos han tratado de reducir esta asimetría, y si bien lo han conseguido en cantidad de datos divulgados no ha sido así en alcance ciudadano. Estudiamos el caso de los datos presupuestarios y las dos principales herramientas de visualización gráfica cuya intención es cerrar esta brecha. Mediante cuestionario respondido por sus responsables en 34 administraciones españolas, tratamos de encontrar respuesta a las principales cuestiones relacionadas con su adopción y uso. Impulso político o consensuado con técnicos, proactividad antes que cumplimiento de normativas de transparencia, desenfoque a la hora de fijar un público objetivo, usos adicionales a los teóricamente esperados y problemas de gobernanza en su operativa son los principales resultados obtenidos.

Palabras clave: datos abiertos; visualización; presupuesto público; España; transparencia; reutilización; ISP - Información del Sector Público.

\section{Online graphical visualisation tools for government open budget data.}

Abstract: The dissemination of public information by governments and administrations is generally too technical and beyond the reach and understanding of most of the citizens to whom they must be accountable and involve in their participatory processes. Progress in open data policies has sought to reduce this asymmetry, and while this has been achieved in terms of the amount of data disclosed, it has not been so in terms of citizen coverage. We studied the case of budget data and the two main graphic visualization tools intended to close this gap. By means of a questionnaire answered by their managers in 34 Spanish public administrations, we try to find answers to the main questions related to their adoption and use. Political impetus or consensus with technicians, proactivity rather than compliance with transparency regulations, lack of focus when setting a target audience, additional uses to those theoretically expected and governance problems in its operation are the main results obtained.

Keywords: open data; visualisation; Spain; transparency; public budget; data reuse; PSI-Public Sector Information.

Copyright: ( $) 2021$ CSIC. Este es un artículo de acceso abierto distribuido bajo los términos de la licencia de uso y distribución Creative Commons Reconocimiento 4.0 Internacional (CC BY 4.0). 


\section{INTRODUCCIÓN}

Multitud de datos de las administraciones públicas se han puesto en los últimos años a disposición de los ciudadanos. Primero a través de la web institucional, y luego a través de portales de datos abiertos o de portales de transparencia. Su razón se ha encontrado en las crecientes demandas de transparencia de los ciudadanos y en el impulso que ha recibido el concepto de gobierno abierto con su énfasis en los datos públicos abiertos u open data como fuente de transparencia, participación y colaboración. Multitud de datos han pasado a estar a disposición de la ciudadanía en formatos digitales reutilizables y con licencia abierta, y con base en ellos terceros ajenos a la administración pública han podido construir aplicaciones, visualizaciones o, simplemente, informaciones.

Los escenarios en los que a las administraciones y gobiernos se les demanda transparencia y rendición de cuentas son tantos como los ámbitos en que desarrollan sus actuaciones y cómo estas intervienen en las vidas e intereses de individuos y organizaciones. Pero de todos ellos, el ámbito presupuestario resulta el más básico y sirve como cimiento de todos los demás. El presupuesto es una herramienta básica de gestión pública que supone la expresión detallada económica, financiera y contable de las políticas desarrolladas por una administración durante un año natural, tanto a nivel de previsión como de ejecución realizada. Es por ello que se encuentra en el corazón del proceso democrático de legitimidad y rendición de cuentas, ya que es la imagen y registro de los recursos que los gobiernos obtienen de los ciudadanos y el uso que hacen de ellos tratando de ser eficientes, eficaces y equitativos y evitando cualquier abuso de poder tanto en las decisiones gubernamentales como en las operaciones de las administraciones públicas como herramientas de política y gestión.

De manera general la rendición de cuentas de los gobiernos se modeliza en dos ramas (O'Donell, 1994): horizontal o vertical. El modelo horizontal son las condiciones que los gobiernos y sus administraciones se imponen jurídicamente a sí mismos, y suponen el control de unos órganos de la administración pública sobre otros; es un control de expertos para técnicos, por tanto, para público reducido, aunque en una democracia representativa supone control y rendición de cuentas indirectos a la ciudadanía. No obstante, su documentación y resultados suelen ser lejanos al ciudadano común y de difícil comprensión dado su tecnicismo. Por el contrario, el modelo vertical es más directo y supone una rendición de cuentas enfocada a la ciudadanía que aprobaría o desaprobaría las acciones gubernamentales mediante el uso de vías participativas y de debate públicos, acciones de protesta u apoyo y, finalmente, el proceso electoral. No obstante, como la práctica demuestra, el proceso vertical necesita también de intermediarios que interpreten y faciliten el acceso a los datos e informaciones. Y aquí es donde la sociedad se está enfrentando de manera exponencialmente creciente a problemas de noticias falsas, manipulaciones y desinformación, que amenazan con pervertir seriamente su normal funcionamiento democrático.

Esta investigación profundiza en el uso de tecnología digital de carácter cívico para facilitar el acceso y comprensión a los ciudadanos de una documentación de carácter técnico como es el presupuesto de las administraciones públicas, que debería ser un elemento de uso frecuente en el debate ciudadano, manejable por los sectores de la población interesados en lo público, comprensible en sus contenidos y totalmente accesible a través de la tecnología digital. Nuestro objetivo es doble: (1) facilitar futuros procesos de implantación de esta tecnología en las administraciones públicas; (2) reenfocar el debate académico sobre divulgación de datos abiertos, en nuestra opinión excesivamente centrado en la simple oferta informativa, incorporando aspectos relativos a los medios técnicos, usos y usuarios. A tal fin, en los siguientes apartados presentaremos una breve exposición teórica respecto a la necesidad y uso de la tecnología cívica de visualización presupuestaria para comunicar información técnica compleja a los ciudadanos más allá del paradigma de los datos públicos abiertos. Posteriormente, formularemos unas preguntas de investigación que consideramos pertinentes para la evaluación de esta tecnología. Finalmente, basándonos en los datos empíricos obtenidos mediante cuestionario entre los responsables de la implementación de esta tecnología, responderemos a las preguntas y estableceremos unas conclusiones que resulten útiles tanto para investigadores como para potenciales responsables de futuras implementaciones de esta tecnología.

\section{MARCO TEÓRICO}

La relación gobiernos-ciudadanos se ha visto marcada en los últimos años por el auge del paradigma denominado gobierno abierto, un nuevo escenario de relación entre gobiernos y ciudadanos basado en la transparencia para fomentar la participación y la colaboración entre administraciones y ciudadanos. Como herramienta básica de comunicación emplea los denominados datos abiertos u open data, persiguiendo alterar el equilibrio de información en favor del ciudadano en la problemática de agencia en que se enmarcan transparencia y 
control (García-García, 2014). Son numerosas las definiciones de lo que supone el gobierno abierto, que ha evolucionado desde el planteamiento inicial de Parks (1957), como una simple apertura pública de información antes custodiada por los gobiernos, a un planteamiento más ambicioso que aúna la tecnología digital con la filosofía del software libre y el poder de Internet para mejorar la innovación, eficiencia y flexibilidad de los gobiernos (Yu y Robinson, 2012).

Clarke y Francoli (2014) afirman que en esta transformación el término gobierno abierto ha devenido en un mutante semántico, siempre relacionado de una manera u otra con transparencia, responsabilidad, rendición de cuentas, participación, mejor gobierno o crecimiento económico, dependiendo del gobierno que invoque el término. Por ello, en la práctica nos enfrentamos a múltiples definiciones de gobierno abierto, y como consecuencia también de datos abiertos para el ámbito presupuestario. Los compendios de ambas definiciones de Clarke y Francoli (2014) y Gray (2015) se centran en sus aspectos legales y técnicos. Muestran coincidencia en las definiciones, pero no así en su aplicación práctica, que se movería entre el alto valor moral de la transparencia financiera y presupuestaria por sí misma, con independencia del método concreto que se aplique para alcanzar este fin, y el valor intrínseco de los datos abiertos como materia prima para potenciales reutilizadores independientemente del uso que se les pueda llegar a dar. De acuerdo con Gray (2015), oscilar hacia un extremo u otro sería una consecuencia de las agendas políticas y su visión de la política fiscal.

En todo caso, la información presupuestaria fue de la primera en ser divulgada por los gobiernos como parte de su estrategia de apertura basada en la Red (Moon, 2002; Cárcaba García y García García, 2003; Torres y otros, 2005; Chen y otros., 2006). Inicialmente, en la década de los 2000 estos datos eran simplemente publicados en las webs institucionales de los gobiernos y administraciones, bien en HTML o en documentos PDF. A medida que avanzaba la siguiente década pasaron a ser divulgados en portales de transparencia o en portales de datos abiertos, en función de que se ofrecieran documentos PDF con el presupuesto íntegro o formatos estructurados bajo licencias libres que permitieran su reutilización, si bien en ocasiones ha primado más el continente tecnológico que el contenido informativo para aparentar avances en transparencia y rendición de cuentas que no se han correspondido con deseos sinceros (García-García y Curto-Rodríguez, 2018). En este sentido, una de las problemáticas asociadas a la divulgación sería el nulo análisis de la demanda de información pre- supuestaria suministrada como datos abiertos y el amplio desconocimiento existente sobre cómo, para qué y con qué fin se usa (Gray, 2015). Este hecho probablemente entronque con el problema de la relación entre transparencia y rendición de cuentas como un problema de caja negra (Meijer, 2013), pues pareciendo evidente una relación entre ambas la literatura académica no ofrece conclusiones generales sobre sus efectos.

No obstante, la verticalidad a la que se enfocan las políticas de gobierno y datos abiertos no es absoluta ni directa hacia el conjunto de los ciudadanos. Tal vez ello sea una causa más del problema definido por Heald (2012) como los missing users (usuarios perdidos), describiendo la diferencia entre las expectativas y el uso real en la divulgación de información pública. El papel del experto como intermediario entre los datos y documentación originales y el público objetivo sigue vigente, tanto mediante los tradicionales medios de comunicación de masas como a través del activismo de datos (Fuchs, 2010; Gutiérrez, 2018) e incluso de formas híbridas entre ellos. Tal y como explica Baack (2015), los activistas de datos han contribuido a reformular las relaciones de agencia entre gobiernos y ciudadanía, mediante la aplicación de los valores del software y cultura libre a la creación y uso de los datos; en este proceso habrían revalorado a los mediadores como necesarios para hacer los datos más accesibles, y contribuido a transformar el periodismo para recuperar su lugar natural de intermediario y contrapoder. Resulta evidente que por mucho que se facilite el acceso a los datos y documentos originales, su plena comprensión y contextualización puede seguir requiriendo de unos conocimientos y disponibilidad de tiempo de los que el ciudadano no dispone. En este sentido, en las dos últimas décadas, con base en la libertad que la Red otorga para las actuaciones coordinadas de ciudadanos, pero también de grupos de interés, han surgido nuevos modelos comunicativos con una capacidad mejorada para la defensa y promoción de puntos de vista particulares y para impulsar el debate político, y que evitan los marcos sociales y organizativos de los medios de comunicación tradicionales. Así es como un proceso inicialmente considerado como positivo por su poder para reducir asimetrías informativas (Benkler, 2011), o al menos no necesariamente negativo, ha empezado a condicionar el modelo vertical de rendición de cuentas y el debate político en general. No obstante, la emergencia de los fenómenos de fake news y desinformación ha puesto en jaque a este proceso.

Por fake news se entienden aquellas informaciones inventadas que replican las formas de divulga- 
ción clásica de los medios de comunicación, pero no sus procesos internos editoriales para asegurar la corrección y credibilidad que en el pasado han tenido gran relevancia en equilibrar libertad y responsabilidad de prensa (Lazer y otros, 2018). Si bien el término ha adquirido una cierta notoriedad, no captura toda la complejidad de las formas de manipulación informativa (Nielsen y Graves, 2017). Por ello, es más riguroso hablar de desinformación, refiriéndose al proceso de generación y divulgación de informaciones erróneas, tendenciosas, falsas o imprecisas y, en particular, cuando éstas se difunden por medios digitales tales como algoritmos propietarios o bots que actúan de agentes de actores políticos tratando de manejar sutilmente a la opinión pública (Woolley y Howard, 2016). De manera general, los algoritmos de las grandes plataformas sociales premian a los usuarios que basan sus contenidos en ruido y emociones fuertes, permitiendo a grupos reducidos posicionarse de manera central en el debate e interrelacionarse mucho más con los grupos de indecisos (Johnson y otros, 2020). La creciente transformación de la Red en un campo de batalla político acentúa el problema de la desinformación y sus negativas consecuencias, especialmente cuando se combina con publicidad personalizada en redes sociales y plataformas de búsqueda según datos personales recogidos de los ciudadanos sin su consentimiento. Por ello, en un mundo interconectado y con una creciente polarización política, la desinformación ha adquirido niveles de complejidad y escala sin precedentes (Wardle y Derakhshan, 2017). Especialmente impulsadas por las casi nulas barreras a la difusión que ha generado la tecnología digital de mensajería y redes sociales, y por el respaldo implícito de los usuarios hacia sus contactos y entorno que suponen los actos de compartición o calificación positiva como me gusta y likes. Por estas vías, la desinformación se difunde más rápidamente y a través de más individuos que las informaciones veraces, particularmente cuando su temática es de carácter político (Vosoughi y otros, 2018) y logra elevarse a la categoría de meme, entendida como una idea de fácil contagio que logra captar la atención y guiar el comportamiento y opinión basándose en las emociones humanas (Rodríguez, 2013).

Además, cuando en este proceso se encuentran cifras, como es el caso de los gastos e ingresos de los gobiernos en sus acciones políticas, juega un importante papel el sesgo cognitivo definido como efecto ancla por Kahneman (2012). Este fenómeno consiste en que la primera cifra recibida ancla el pensamiento y condiciona a ella el futuro juicio y acción del receptor, con independencia de su veracidad. Por ello, en un entorno de sobrecarga informativa, que los gobiernos y administraciones ofrezcan datos e informaciones de una forma sencilla, accesible y contextualizada es prácticamente un requisito para lograr los objetivos de transparencia y rendición de cuentas, tal y como describió el estudio de Worthy (2015) al respecto del impacto de los datos abiertos en Reino Unido. Así, uno de los muchos usos que se les ha dado a los datos abiertos ha sido crear visualizaciones que faciliten su comprensión y contextualicen sus contenidos. En esta misión, los gobiernos fueron manifiestamente superados por el activismo de datos que se fue desarrollando paralelamente a la divulgación de datos por los gobiernos. Estos activistas, individuos u organizaciones, no sólo desarrollaron contenidos en base a los datos, sino también herramientas digitales que distribuyeron como software libre y que permitieron revelar el valor cívico de los datos superando formas de exclusión y permitiendo a la ciudadanía tomar sus propias decisiones y actuar en consecuencia, pues el acceso a la información no permite sólo tener conocimiento sino usarlo en el propio interés (Gutiérrez, 2018). Sin embargo, a pesar de estas experiencias debemos evitar caer en la trampa del solucionismo tecnológico (Morozov, 2013) y tener presente que la mera aplicación de la tecnología digital a un problema no es necesariamente la solución de este, sino que deberemos conocer todo el marco de referencia y acción en el que se circunscribe. Asimismo, tampoco debemos caer en la trampa de que para ejercer un derecho ciudadano no baste con tenerlo reconocido, sino que se haya de utilizar un elemento tecnológico sin el cual su ejercicio deviene imposible (de la Cueva González-Cotera, 2019), por lo que las soluciones de visualización han de entenderse como un añadido a todos los derechos históricamente reconocidos respecto a la transparencia y rendición de cuentas.

Las herramientas de visualización presupuestaria permiten explorar fácilmente los presupuestos públicos y comprender y contextualizar los gastos e ingresos que definen las políticas y actuaciones de las distintas administraciones públicas. Todas estas visualizaciones generan una vista global de los gastos y los ingresos a partir de la cual se puede ir descendiendo jerárquicamente de mayores a menores niveles de detalle y examinar la participación sobre el total, así como su evolución temporal. Es especialmente destacable que, aunque sea facilitado en la descarga de los datos, se abstrae parcialmente la complejidad de la información técnica presupuestaria centrando la visualización en políticas (estructura económica y funcional del presupuesto: cómo se gasta y en qué se gasta) en vez de en órganos de ejecución presupuestaria 
(estructura orgánica). Resulta evidente que este tipo de herramientas gráficas facilitan el examen, la comparación y la contextualización de la información a personas con menores conocimientos en materia presupuestaria. A modo de ejemplo, pueden consultarse los Presupuestos Abiertos del Ayuntamiento de Madrid [1] o los Datos Económicos de la Diputación de Castellón [2].

En el caso de los conjuntos de datos abiertos, el grado de reutilización que permiten de la información es mayor, pero requieren de un cierto conocimiento de herramientas informáticas de tratamientos de datos. Los datos abiertos tienen como objetivo facilitar su reutilización para elaborar informaciones o visualizaciones más complejas, y presuponen que el usuario que accede a ellos tiene conocimientos presupuestarios, pero también sobre herramientas de software para poder operar con ellos. Por el contrario, la visualización de información permite que datos complejos puedan cobrar sentido, y como complemento a las representaciones textuales o verbales ofrecen un amplio potencial para servir de apoyo al conocimiento y a la comunicación en temáticas complejas como son los presupuestos de las administraciones publicas. Su objetivo principal está en facilitar la comprensión a personas no versadas en la materia de las cantidades consignadas para determinadas políticas y partidas mediante una comparativa con otras políticas y partidas del mismo periodo o anteriores a través de su evolución. No obstante, ambas formas de divulgar son preferibles al clásico documento PDF que exige de conocimientos presupuestarios para su comprensión, no permite un sencillo examen, comparación o contextualización, ni además permite su tratamiento con herramientas digitales para extraer datos y construir informaciones.

Por todo ello, con base en la literatura expuesta, nos planteamos cinco preguntas de investigación (ver tabla 1) que consideramos básicas para orientar cualquier investigación posterior sobre el uso de herramientas de visualización de la información presupuestaria en procesos de gobierno abierto, así como su implantación práctica en administra- ciones públicas. Su respuesta nos permitirá conocer para la fase de planteamiento del proyecto implantación de quién parte la iniciativa, con qué objetivos y si han existido barreras relevantes técnicas o económicas; y para la fase de uso quiénes han sido los usuarios reales respecto a los inicialmente planteados y si existen riesgos que amenacen la continuidad del proyecto.

\section{METODOLOGÍA}

La investigación se ha centrado las dos principales herramientas de visualización en uso en las administraciones españolas. La primera es "Presupuesto Abierto de Aragón - Aragón Open Budget", la más extendida entre las administraciones públicas españolas. La aplicación fue producida en el marco del proyecto Aragon Open Data [3] con licencia de software libre European Union Public Licence 1.1 que otorga libertad de uso, reutilización, adaptación y modificación con la única condición de mantener el código fuente resultante también como software libre. Con frecuencia, esta aplicación se encuentra también denominada como "Dónde Van Mis Impuestos" (DVMI), nombre empleado para la herramienta por la Fundación Ciudadana Civio, que fue su desarrollador para el proyecto Aragon Open Data y que realiza la mayor parte de las adaptaciones para las administraciones españolas [4]. Esta última denominación es la que generalmente se encuentra en las webs institucionales para la herramienta, combinada con otras como por ejemplo "Cuentas Claras" o "Presupuestos Visuales". La determinación de la población objeto de estudio se centró en la herramienta con independencia del nombre que recibiera por parte de la administración, quedando integrada por las 32 administraciones públicas españolas que en mayo de 2019 la tenían en uso en su web institucional y la mantenían actualizada ( 6 comunidades autónomas, 24 ayuntamientos y 2 consejos insulares). La cobertura de la herramienta sería de cerca de 17 millones de habitantes, algo más de un tercio de la población española, que podían emplear la aplicación para visualizar los presupuestos de sus administraciones locales y/o autonómicas.

Tabla 1: Preguntas de investigación

\begin{tabular}{|l|c|l|}
\hline \multicolumn{1}{|c|}{ FASE } & \multicolumn{2}{c|}{ PREGUNTA DE INVESTIGACIÓN } \\
\hline \multirow{3}{*}{ Planteamiento } & I & ¿De quién parte la iniciativa para la implantación? \\
\cline { 2 - 3 } & II & ¿Cuáles son los objetivos perseguidos con la herramienta? \\
\cline { 2 - 3 } & III & ¿Existen barreras tecnológicas, financieras u organizativas? \\
\hline \multirow{2}{*}{ Uso } & IV & ¿Coinciden los usuarios definidos a priori con los reales? \\
\cline { 2 - 3 } & V & ¿Existen riesgos que puedan llevar al abandono del proyecto? \\
\hline
\end{tabular}


La segunda herramienta en estudio es el visor "Con Mis Impuestos" (CMI) desarrollado por la empresa española Idi Eikon para su plataforma de servicios de gobierno abierto "Governalia". En este caso nos encontramos ante un software no libre sino privativo, pero con funcionalidad muy similar a DVMI. Pero a diferencia de este, el uso de CMI se encuentra mayoritariamente concentrado en el entorno geográfico de la costa mediterránea española en administraciones generalmente de menor tamaño. La población en estudio comprendió a las 24 administraciones públicas españolas que en mayo de 2019 tenían la herramienta CMI en uso en su web institucional y la mantenían actualizada (2 diputaciones provinciales, 1 consejo insular y 21 municipios). La cobertura de CMI es de cerca de 2,7 millones de habitantes, aproximadamente el $6 \%$ de la población del país.

Como herramienta de investigación se empleó un cuestionario con preguntas estructuradas, principalmente escalas Likert o respuesta múltiple o respuesta abierta cuando procediera. Estas preguntas fueron construidas sobre la base de las preguntas de investigación expuestas en el apartado anterior y de las principales teorías de adopción de tecnologías de la información. La discusión de estas teorías excede el ámbito de este artículo, pero para el lector interesado en sus características y evolución recomendamos la revisión crítica de Tarhini y otros. (2015). El cuestionario se dirigió a los responsables de la aplicación de visualización, definidos como aquellas personas con capacidad de decisión y responsabilidad sobre la herramienta, su implantación, mantenimiento, actualización o retirada. A lo largo del mes de mayo de 2019 el cuestionario se hizo llegar al responsable de cada administración por correo electrónico facilitando una URL para la recogida de datos $y$, posteriormente, por correo postal a aquellas entidades de las que no se hubiera obtenido respuesta tras un recordatorio. La recogida de respuestas finalizó días antes de la formación de los nuevos gobiernos surgidos de las elecciones del 26 de mayo de 2019, evitando un posible sesgo de respuesta por la llegada de nuevos responsables.

La tasa de respuesta fue en total de 38 entidades, compuesta de la totalidad de las comunidades autónomas y más de la mitad de los municipios en estudio. Desglosada por herramientas, la respuesta fue del $75 \%$ para DVMI (24 de 32 administraciones: 6 comunidades autónomas y 18 municipios) y del 58,33\% para CMI (14 de 24 administraciones: 2 diputaciones provinciales, 1 consejo insular y 11 municipios). Creemos que la diferencia en las tasas de respuesta es debida principalmente a la diferencia de tamaño de las distintas administraciones, debido a que los adoptantes de CMI son mayorita- riamente administraciones de menor tamaño comparadas con las adoptantes de DVMI. Esta idea se ve reforzada por el pleno en la tasa de respuesta de comunidades autónomas, diputaciones provinciales y municipios de mayor tamaño, tanto para DVMI como para CMI.

Para las respuestas basadas en escalas Likert, se ha empleado la mediana interpolada como medida de tendencia central (Young y Veldman, 1972; Kiess y Green, 2009). En todos los casos se ha tenido en cuenta la dispersión de los resultados a través de la desviación típica y rango intercuartílico.

\section{RESULTADOS}

En este apartado ofrecemos los resultados obtenidos para las preguntas de investigación planteadas.

\section{1 ¿De quién parte la iniciativa para la im- plantación?}

Una de las principales cuestiones de la investigación era quién había ostentado el liderazgo de la iniciativa de implantación de la herramienta. A priori, pareciera situarse tanto en el área tecnológica como en el área de gestión presupuestaria y financiera; sin descartar que surgiera de una autoridad política en la cúspide de la organización o fuera fruto del impulso de algún empleado sin excesiva capacidad jerárquica. De manera mayoritaria, las respuestas para DVMI sitúan el origen el proyecto en el ámbito del responsable político de la administración, seguido ya a gran distancia de un impulso consensuado entre los diferentes roles citados en la pregunta. En el caso de CMI ambas opciones aparece igualadas como origen de la iniciativa, probablemente de nuevo debido al menor tamaño de las entidades, lo que facilitaría los consensos. Resulta sorprendente, tanto en un caso como en el otro, el escaso origen en las áreas técnicas de tecnologías de la información y comunicación (TIC) o de gestión.

Como principal motivador de este impulso se encuentra el deseo de excelencia, seguido en menor medida por el anhelo de pertenecer a un grupo de entidades innovadoras. Sorprende la escasa tasa de respuesta que obtuvo como motivador el deseo de responder a las peticiones ciudadanas de mejorar la transparencia y rendición de cuentas de la administración pública. No obstante, el reparto de las respuestas es desigual entre ambos grupos, para el caso de DVMI el consenso es muy elevado para el deseo de excelencia, pero la divergencia aumenta en el grupo de CMI donde la tasa de respuesta es ligeramente más elevada para el deseo de pertenecer a un grupo de entidades innovado- 
Figura 1. Ámbito de iniciativa del proyecto

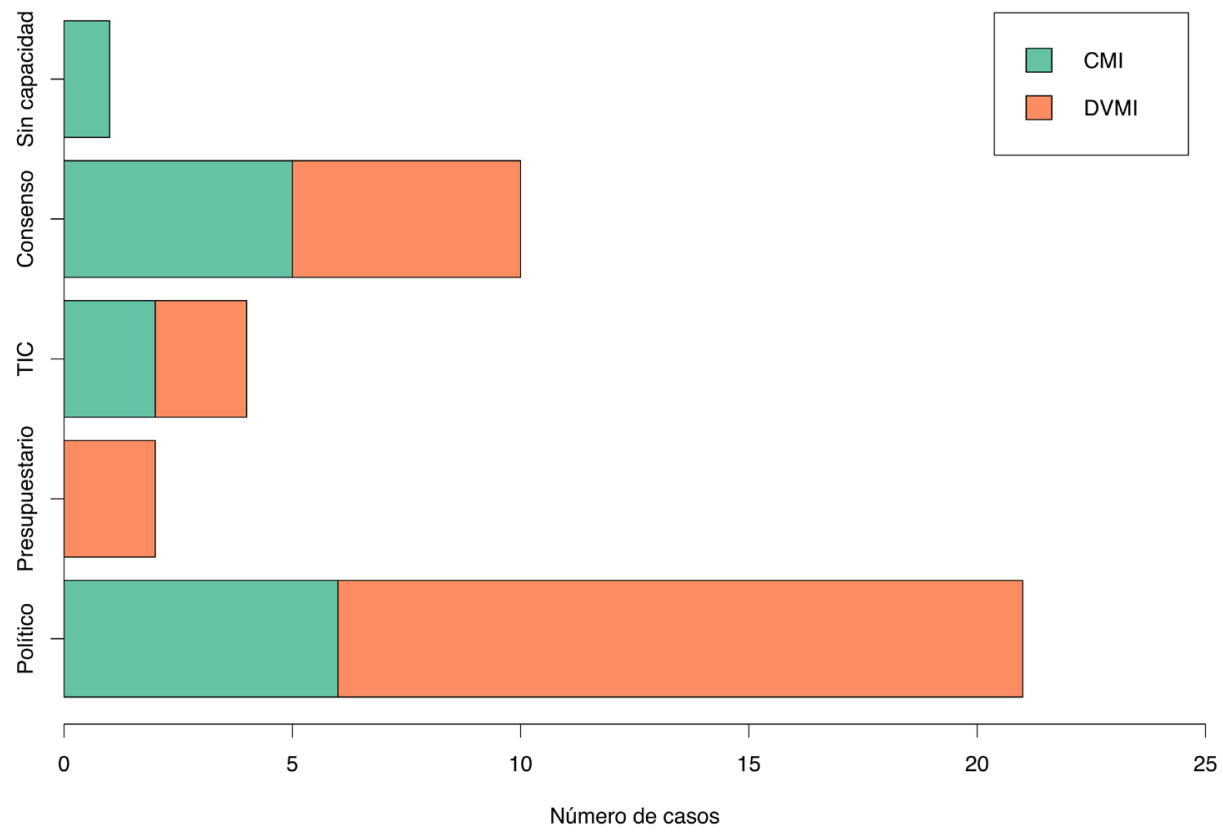

Figura 2. Motivación para el impulso (valoración media)

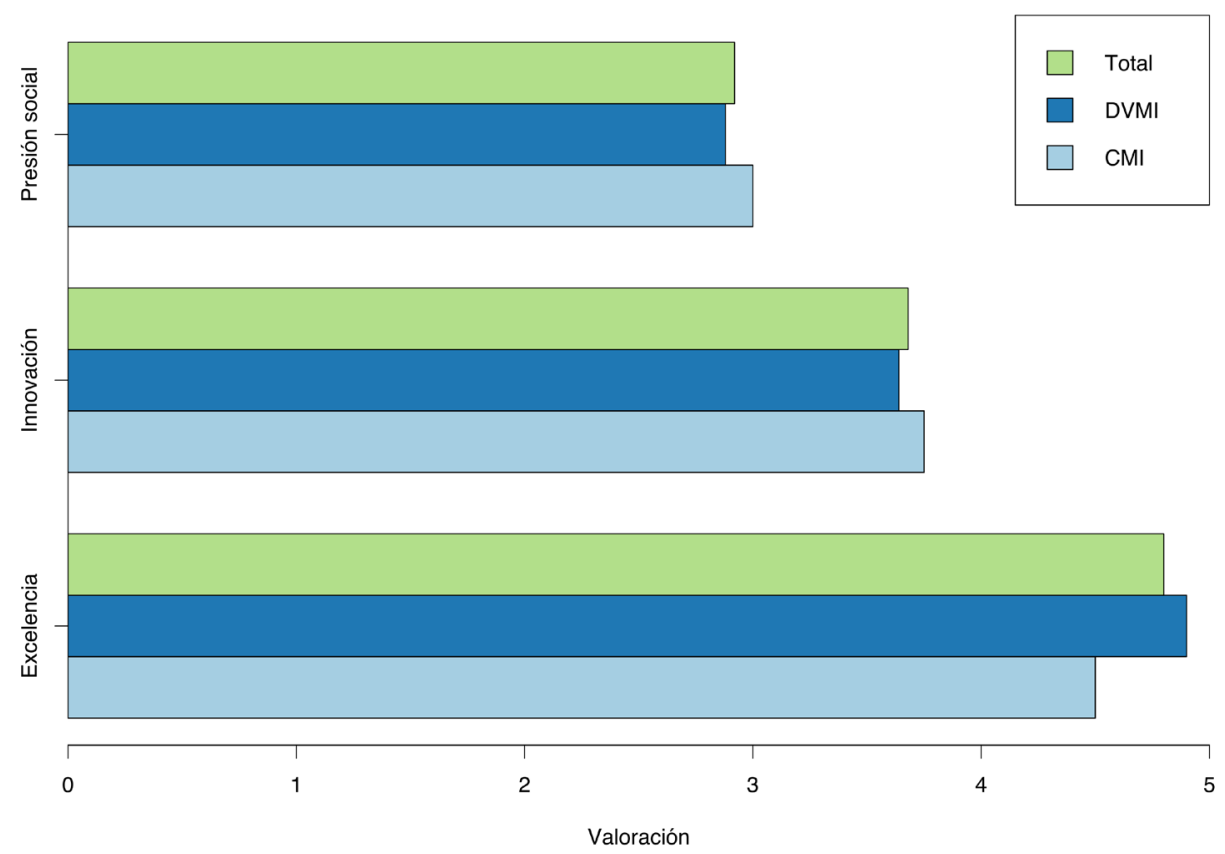

ras e incluso para la respuesta a la demanda social. Consideramos que esta diferencia puede ser debida al hecho de que CMI se comercializa como producto integrante de un paquete de herramientas de gobierno abierto, mientras que DVMI es un producto único. El deseo de mejorar en la carrera profesional no es considerado como un motivador, ni en tasa de respuesta ni por los comentarios recibidos a las preguntas, resultado coherente con que el liderazgo del proyecto no provenga principalmente del cuerpo de funcionarios de TIC o de gestión presupuestaria. 


\section{2 ¿Cuáles son los objetivos perseguidos con la herramienta?}

Mayoritariamente los responsables valoran como primer objetivo de la herramienta la transparencia financiera y presupuestaria, en un grado manifiestamente superior para DVMI que para CMI, un resultado evidente hablando de un visor presupuestario. Pero, también se obtienen tasas de respuesta considerables refiriendo el uso de la aplicación en procesos participativos y en lo que se llama microtransparencia (localización de datos específicos de interés). Este último objetivo es más elevado en el caso de CMI que en el de DVMI, creemos que debido al menor tamaño de estas administraciones que ocasiona una mayor cercanía al ciudadano y que estos se preocupen más de actuaciones concretas. Adicionalmente, en la respuesta abierta para otros objetivos, también se comentan los usos en campañas de prensa y de concienciación fiscal de los ciudadanos. Es evidente que una herramienta puede no tener un único objetivo, pero los resultados muestran como lo que pudiera parecer un objetivo obvio (transparencia financiera y presupuestaria) pudiera no serlo tanto, fijándose otros ya desde su inicio. En particular, la transparencia participativa y la microtransparencia, que pueden ser considerados usos más sofisticados pues conectan directamente con los objetivos de participación y colaboración del gobierno abierto, estarían en línea con la proactividad requerida de gobiernos innovadores.
Asimismo, constatamos que, mayoritariamente y con un amplio consenso, los responsables creen que, además de visualmente, los datos deben poder ser accesibles en formato de datos abiertos, esto es, con licencia libre para su reutilización y en formato estándar. Por supuesto, siendo datos que respondan a una demanda ciudadana y se publiquen en un tiempo oportuno. En este caso, los encuestados creen que ellos se preocupan más de este aspecto que su propia entidad.

También resulta de interés conocer si la razón de que se establezcan los objetivos se debe a un deseo de tener una herramienta que permita cumplir con el ordenamiento jurídico respecto a la publicidad de información presupuestaria, o por el contrario a una política proactiva de transparencia hacia el ciudadano, facilitando el acceso a los datos con independencia de que se ofrezcan en otros medios y formatos suficientes para cumplir las obligaciones legales. Las respuestas fueron abrumadoramente coincidentes en el afán de proactividad sobre el cumplimiento de la normativa legal, si bien en el caso de CMI la diferencia es más estrecha, de nuevo creemos que debido al menor tamaño de las entidades. Resulta obvio que cumplir con los requisitos legales de publicidad puede hacerse con simples documentos PDF o incluso con conjuntos de datos abiertos, pero implementar este tipo de herramientas de visualización es ir más allá de lo requerido por cualquier ordenamiento jurídico actual.

Figura 3. Objetivos de transparencia

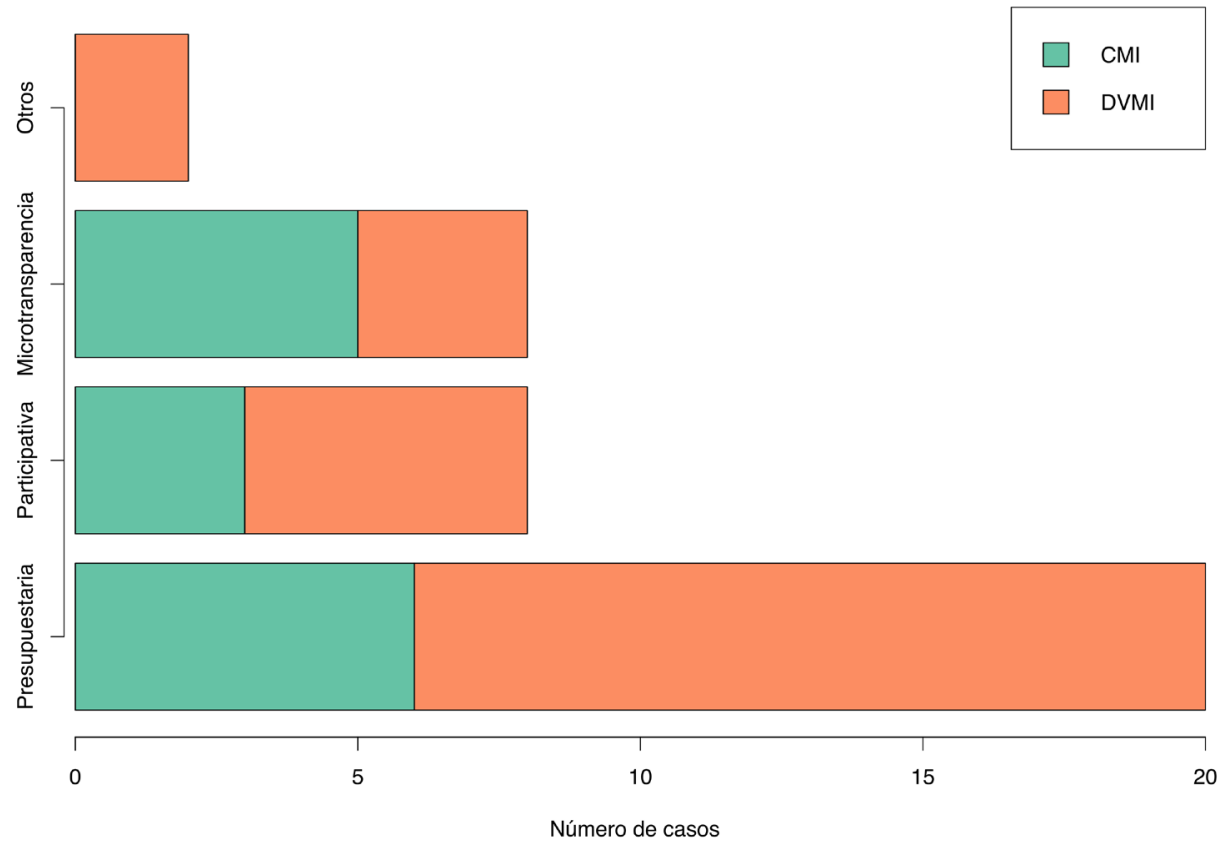


Figura 4. Marco regulatorio y proactividad (valoración media)

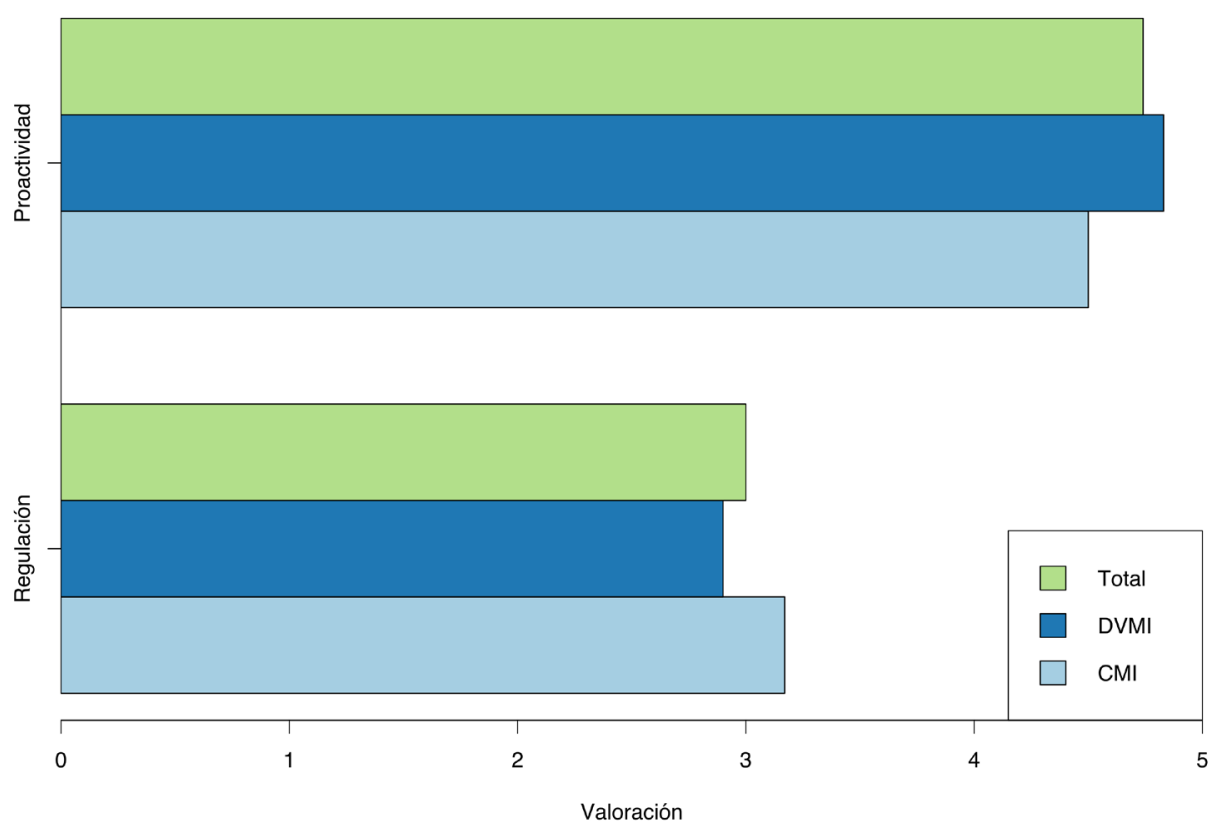

Figura 5. Barreras (valoración media)

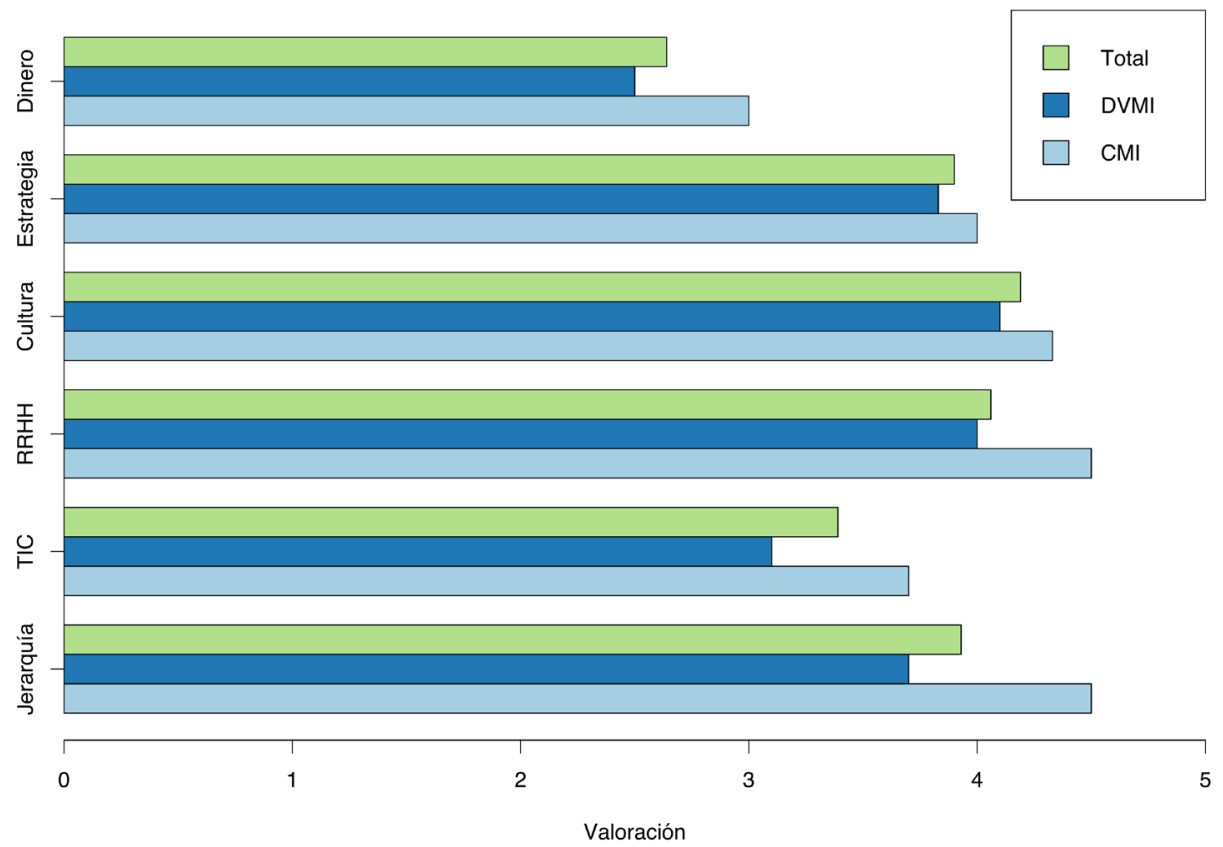

\section{3 ¿Existen barreras tecnológicas, financie- ras u organizativas?}

Respecto a la existencia de barreras económicas o tecnológicas que pudieran haber dificultado el proyecto, no se consideraron en ningún momento muy relevantes $y$, en particular, las de carácter económico obtienen la menor valoración.
Reciben una mayor valoración otro tipo de barreras intangibles, principalmente las dinámicas organizativas entendidas como las formas habituales de hacer las cosas, seguidas de falta de recursos humanos para la actualización y mantenimiento de la herramienta, la falta de estrategias claras de transparencia y rendición de cuentas, e 
incluso la resistencia de superiores jerárquicos. En nuestra opinión, estos cuatro aspectos formarían parte del mismo problema: acciones que se ponen en marcha para reforzar la imagen de la administración como transparente, sin un plan preestablecido para canalizar recursos de una manera sostenida que permita alcanzar unos objetivos claramente definidos. No obstante, hacemos notar que en las respuestas existe una falta de consenso bastante elevada para estas barreras, y que las administraciones del grupo CMI, aunque mantienen la jerarquía entre ellas, otorgan una mayor puntuación a todas las barreras, creemos que de nuevo debido al menor tamaño que hace que el proyecto sea relativamente más complejo de llevar a cabo.

No se consideró excesiva la dificultad técnica de implantación, siendo esta consideración aún más favorable en el caso de DVMI, si bien aproximadamente el $80 \%$ de las administraciones recurrieron a contratación con terceros. Tampoco la diferencia de licencia del software, libre para DVMI y privativa para CMI, parece haber actuado como barrera, aunque en el caso de DVMI se encuentra una mayor valoración positiva a que sea software libre sobre la valoración de CMI como producto privativo. En ambos casos los responsables subrayan las potencialidades de mejora, ampliación o personalización futura sobre cualquier coste económico de adquisición y puesta en marcha.

\section{4. ¿Coinciden los usuarios definidos a priori con los reales?}

Los gestores de la herramienta fueron preguntados por los grupos de usuarios más interesados en los datos que suministra. De manera un tanto sorprendente, por tratarse de una herramienta de tecnología cívica teóricamente al servicio del ciudadano, los ciudadanos individuales fueron considerados el grupo con menor interés en los datos presupuestarios y de manera más acusada en el grupo CMI; por el contrario, los grupos políticos fueron considerados los grupos de mayor interés, seguidos por los medios de comunicación. Otros grupos como contratistas con la administración y asociaciones obtuvieron valoraciones muy cercanas. El resultado es consistente con el modelo de rendición de cuentas vertical, con los grupos políticos y medios de comunicación actuando como intermediarios entre ciudadanos y gobiernos. Sin embargo, resulta difícil entender el porqué de la valoración recibida por los grupos políticos, normalmente considerados estrechamente ligados a la acción gubernamental o de oposición y, por tanto, potencialmente sesgados en sus interpretaciones, si bien es cierto que los datos presupuestarios son la materia prima de su trabajo. El consenso en esta calificación resultó muy elevado, con desviaciones muy similares para todos los grupos, con independencia de la herramienta empleada, resultando aún mayor para la valoración de los grupos

Figura 6. Usuarios (valoración media)

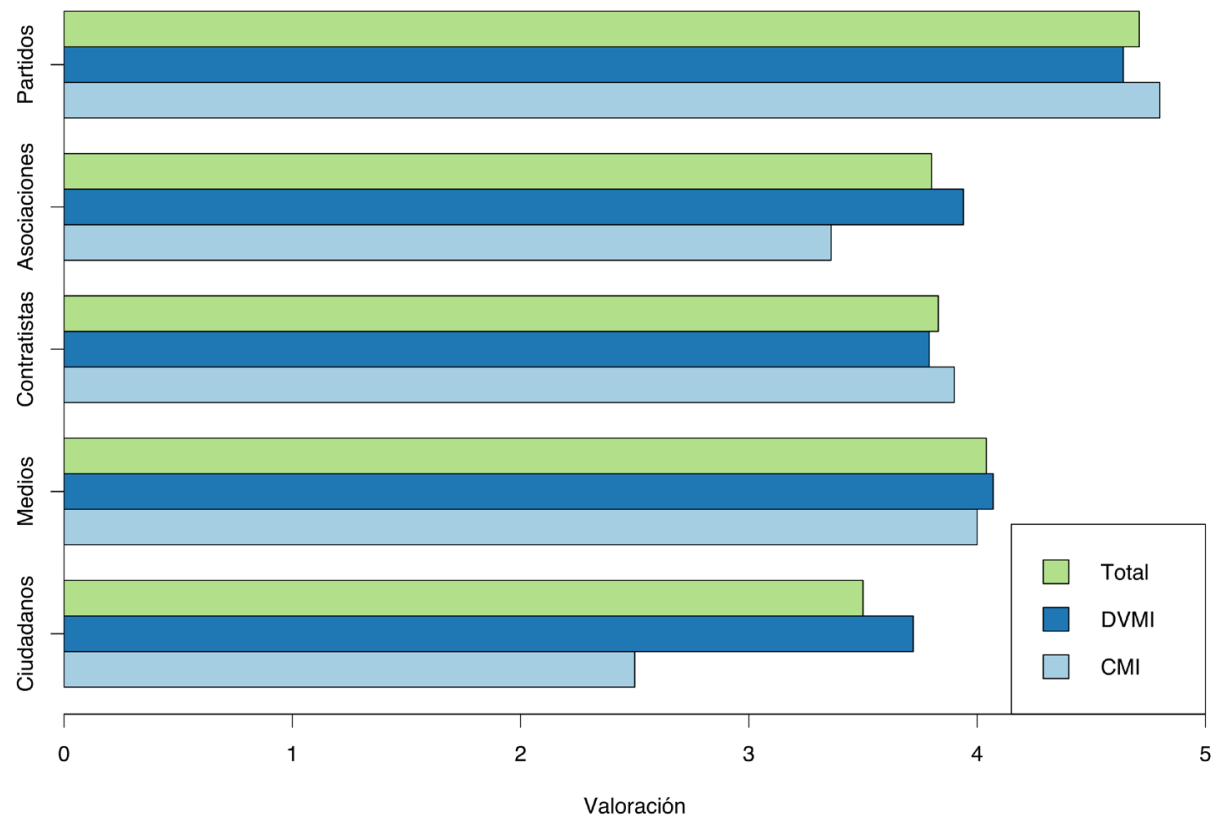


políticos en el caso de administraciones con la herramienta CMI.

Preguntados por los usos concretos, mayoritariamente se refirieron a usos en informaciones e investigaciones periodísticas. En cambio, resultaron más escasos otros que, a priori, también pudieran resultar interesantes, como el uso en procesos participativos o el uso para solicitar explicaciones a los responsables políticos sobre acciones de gobierno o gasto público. El resultado es congruente con el obtenido para los usuarios, particularmente en el caso de los medios informativos, y también, como se mostrará más adelante, con los objetivos de transparencia perseguidos. Destacamos también la mayor valoración en el grupo CMI para los usos internos y participativos, lo que refuerza nuestra idea de que el tamaño resulta relevante para el planteamiento y uso de estas herramientas, quizá buscando un rendimiento adicional a la inversión efectuada en software.

Los resultados obtenidos a esta pregunta de investigación nos parecen en especial destacables, especialmente cuando observamos los comentarios recibidos en las preguntas abiertas al respecto. Los responsables coinciden en achacar el escaso uso ciudadano a la falta de conocimientos básicos sobre el presupuesto y creen que su impacto en la transparencia y rendición de cuentas podría haberse potenciado acompañando su implantación con campañas informativas acerca de su existencia, modo de empleo y ejemplos de uso y obtención de datos. Por ello, no les resulta extraño que la mayor parte de los usos procedan de medios informativos o grupos políticos. Adicionalmente, las preguntas de respuesta abierta nos facilitaron un uso no previsto a priori, el uso por parte de la propia administración para sus informes internos sobre asignación presupuestaria y comparativas interanuales, y la generación de recursos gráficos para campañas informativas en los medios sobre las acciones de gobierno. Este tipo de usos aparecen referidos en las respuestas casi tanto como su uso en informaciones periodísticas.

Queremos incluir aquí también un comentario recibido de varios responsables: el valor añadido de la herramienta como un punto de encuentro entre los desafíos que para los usuarios puede suponer el procesamiento de datos abiertos (formatos de texto estructurado, CSV) y la poca versatilidad de los documentos PDF para su examen y análisis. Así, la herramienta quizá no debería focalizarse tanto a grupos de usuarios como los antes mencionados sino a grupos de usuarios con una necesidad intermedia de procesado de los datos.

\section{5 ¿Existen riesgos que puedan llevar al abandono del proyecto?}

Con esta pregunta de investigación hemos querido responder a cuáles son los potenciales peligros que afectan a la herramienta una vez instalada y/o

Figura 7. Principales usos (valoración media)

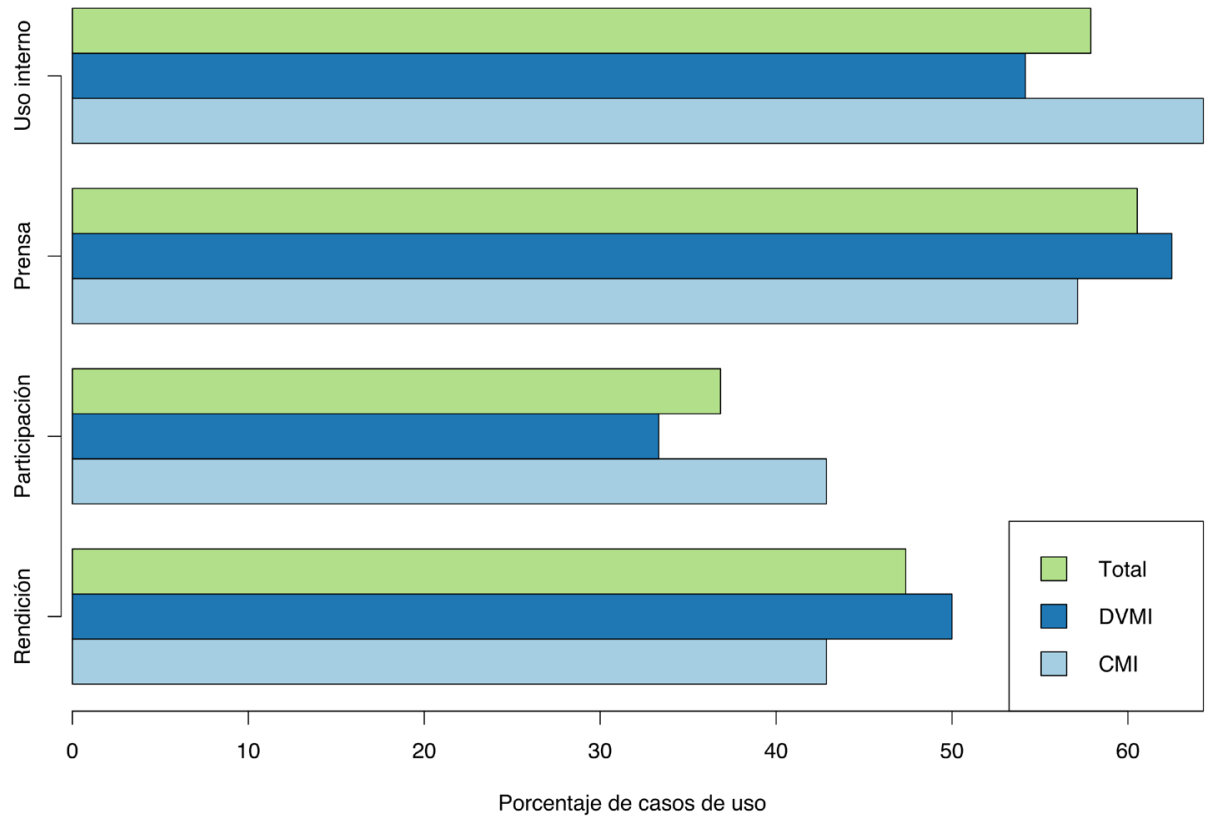


que puedan condicionar su expansión a otras administraciones con base en la experiencia de los pioneros.

De acuerdo con las respuestas abiertas recibidas de los responsables, la transferencia de datos desde el software de gestión contable y presupuestaria a la aplicación de visualización supone el mayor problema al que se enfrentan, debido a la cambiante estructura presupuestaria por reorganizaciones administrativas. Esto supone tener que dedicar tiempo de recursos humanos a esta tarea para poder mantener la comparabilidad interanual. En torno a esta problemática también se refirieron con frecuencia problemas ocasionados por la falta de una estrategia clara en temas de transparencia. Concretamente, la falta absoluta de procedimientos establecidos que determinen quién, cómo y cuándo debe ocuparse de solucionar el problema de casación de partidas presupuestarias reasignadas entre órganos y funciones en sucesivos ejercicios económicos o de actualizar los datos. Curiosamente, las fases previa y posterior a estos problemas (estas son, la recolección de datos y su posterior carga en la aplicación una vez depurados y reclasificados) fueron las consideradas como las partes de menor dificultad técnica.

\section{CONCLUSIONES}

Las herramientas de visualización presupuestaria rebajan los niveles de conocimiento requeridos de los ciudadanos para acceder, comprender y contextualizar la información presupuestaria. Por ello, son un instrumento óptimo para la rendición de cuentas vertical a los ciudadanos, sin perjuicio de los canales clásicos formales. Nuestra investigación recogió datos por medio de un cuestionario a los responsables de las dos principales herramientas empleadas por las administraciones españolas. La tasa de respuesta global del $75 \%$ nos permite afirmar la bondad de nuestros resultados, así como su capacidad de orientación para futuras experiencias de implantación.

Los resultados nos muestran un bajo interés de la ciudadanía por los datos presupuestarios abiertos, así como la necesidad de disponer de intermediarios o de desarrollar interfaces que permitan el acceso a los datos y su contextualización. También coinciden con la literatura en la no existencia de un usuario medio de los datos, sino en grupos con interés muy específico en determinadas informaciones particulares (Gray, 2015; Worthy, 2015), lo que hace que el uso de la herramienta sea muy inestable y dependiente de las cambiantes necesidades informativas de esos grupos. El escaso número de usuarios no debe identificarse nece- sariamente con escaso interés en los datos, sino con la todavía existente necesidad de disponer de expertos que puedan crear y contar una historia al público en base a los datos; por ello, aunque estos datos sean mucho más accesibles que en la era predigital o en los años tempranos de la divulgación en línea, es todavía necesario que los datos sean obtenidos, contextualizados, interpretados y canalizados hacia las personas legas en la materia. Esto implica una inversión en tiempo y recursos que el ciudadano, destinatario final de la rendición de cuentas, no puede enfrentar solo ( $y$, quizá, tampoco debe) y para lo que necesita de intermediarios, normalmente periodistas y otros grupos ciudadanos que realizan las mismas funciones y canalizan los datos ya procesados como información hacia los medios de comunicación. Por ello, no debemos calificar a los escasos usuarios como un fracaso de la herramienta sino simplemente señalar que probablemente el foco y las expectativas se han puesto en el grupo de usuarios equivocado, lo que puede contribuir a explicar el fenómeno de caja negra (Meijer, 2013). A pesar de ello, el software estaría cumpliendo con su objetivo de crear nuevas vías de acceso a los datos que de otro modo precisarían de un mayor número de expertos en la cadena de obtención y contextualización, reduciendo así el uso de los denominados brokers de información (Heald, 2003) y rebajando las asimetrías informativas. Como afirman Royo-Montañés y Benítez-Gómez (2019), para que los datos abiertos sean un catalizador de cambio social no es suficiente con divulgarlos en portales de datos abiertos, sino que también se deben profundizar todos los aspectos que fomentan su utilización para un público no experto. Por ello, tampoco ha de olvidarse el papel de estas tecnologías cívicas en el impulso de la dimensión política ciudadana de conceptos aparentemente corporativos como el de smart-city, donde pueden estar al servicio del empoderamiento ciudadano en el derecho del acceso a la información y en procesos de toma de decisiones participativos, abiertos y transparentes; particularmente en el ámbito de los gobiernos más cercanos a los ciudadanos (Charnock et al., 2019).

También destacamos las brechas de expectativas detectadas. De manera general, los responsables de las herramientas creen que ellos se preocupan más que sus administraciones por atender a la demanda ciudadana de información, por la reusabilidad de los datos y por su oportunidad temporal. Ello es consistente con lo descrito en la literatura de que muchas iniciativas de datos abiertos existen y se mantienen gracias al esfuerzo de personas concretas más allá de cualquier estrategia o programa de gobierno abierto o rendición de cuentas 
(García-García y Curto-Rodríguez, 2019), poniendo la carga de estas iniciativas más en factores individuales que colectivos. La segunda brecha de expectativas es la creada entre los usuarios teóricos (ciudadanos) y los usuarios reales (medios de comunicación y grupos políticos), que pudiera resultar en que no se vieran satisfechos indicadores de éxito preestablecidos, ralentizando o impidiendo la difusión de estas herramientas a otras administraciones. Todo esto, unido a la dificultad operativa de conciliar la cambiante estructura presupuestaria con las estructuras de datos requeridas por las herramientas, y con la declarada aspiración de buscar la excelencia, refuerza nuestro temor de que existe un serio riesgo de amenaza vital para el futuro de las herramientas de visualización presupuestaria.

En un sentido positivo, destaca la independencia de la herramienta de los condicionantes financieros o tecnológicos, en el caso de DVMI en parte por ser software libre y por las expectativas de continuar con mejoras y adaptaciones particulares por cuenta propia, y tanto en el caso de CMI como DVMI por ser la inversión de puesta en marcha generalmente pequeña. No obstante, no queremos dejar de destacar la implementación en algunos municipios de menos de 15.000 habitantes para los que la inversión sí resulta significativa y constituye una clara apuesta por la transparencia y rendición de cuentas. En este sentido, nuestros resultados apuntan a distintos planteamientos en la adopción y uso de estas herramientas según el tamaño, algo que debemos considerar como normal dada la cercanía al ciudadano en las administraciones de menor tamaño y su menor capacidad de recursos que les puede llevar a optimizar los usos del software ya disponible.

El impulso mayoritariamente político a la implementación también es otro de los resultados que merece una reflexión, especialmente unido a la brecha de expectativas sobre los usuarios, ya que desconocemos hasta que punto la adopción de una tecnología cívica puede resultar influenciado por modas. Así, podríamos encontrarnos no tanto ante el deseo de ser transparentes ante la ciudadanía como ante la réplica de experiencias que hayan recibido una sustancial atención mediática cuando fueron puestas en marcha en otras administraciones, con independencia de cualquier fijación de objetivos y evaluación de resultados. Entonces, la adopción de la herramienta se convertiría en un caso particular del fenómeno conocido como openwashing (Heimstädt, 2017). En cualquier caso, dado el reducido coste de la implementación y la relevancia de los datos ofrecidos, creemos que es algo que siempre debería de existir, lo que nos lleva a plantearnos las razones por las que tan pocos gobiernos ofrecen este tipo de visualizacio- nes en sus webs institucionales. En este punto, no podemos sino coincidir con González-Limón y Rodríguez-Ramos (2019) en el positivo papel de la regulación de la transparencia y de la información pública para la aparición de reutilizaciones prácticas de los datos abiertos, aunque siga existiendo una gran carencia en la difusión, si bien creemos que, como muestran los resultados, unos responsables públicos proactivos en busca de la excelencia pueden actuar como un complemento positivo a lo dispuesto en las diferentes normativas.

Los resultados también revelaron la existencia de uno de los problemas emergentes para los expertos en datos públicos abiertos, que se manifiesta cuando la publicación de datos se extiende en volumen y en el tiempo: su gobernanza, definida como la interacción de reglas, estándares, herramientas, principios, procesos y decisiones que influyen sobre qué datos son liberados, cómo y por quién (Brandusescu y otros 2019). Este fenómeno se nos manifestó claramente cuando en los comentarios a las preguntas, reiteradamente los responsables mencionaban la problemática de homogeneizar la cambiante estructura de los presupuestos entre años para permitir las comparativas. En general, no parecían existir procedimientos claros sobre cuándo debería realizarse esta tarea, si debiese formar parte del proceso presupuestario en sí mismo, o quién y como debería ser el responsable de llevarla a cabo. Nos encontramos ante un problema de gobernanza que en el largo plazo pudiera comprometer el uso de estas herramientas o actuar como una barrera a su expansión a nuevas administraciones, pues realmente constituye un coste oculto pagadero en tiempo de recursos humanos. $Y$, generalmente, todas las manipulaciones manuales de datos o manipulaciones con software no específico requieren de tareas repetitivas de escaso valor añadido que con el paso del tiempo tenderán a ser descuidadas y abandonadas a medida que el impulso inicial del proyecto decae (Abella y otros, 2019). En consecuencia, buena parte de los deseos de proactividad y excelencia unidos a estos proyectos deberían enfocarse a solventar este problema de gobernanza. Los adoptantes de herramientas de visualización presupuestaria deberían extender sus estrategias de transparencia y rendición de cuentas a los procesos operativos en torno a ellas, desarrollando, si fuera necesario, nuevas rutinas y procedimientos e incluso aplicaciones de software para reducir o erradicar estos costes ocultos.

En resumen, las aplicaciones de visualización presupuestaria son una tecnología cívica innovadora que, debido a su capacidad para reducir las asimetrías informativas, debería extenderse entre las distintas administraciones públicas. Sin embargo, 
deberíamos evitar caer en la trampa del tecnofetichismo otorgando a las tecnologías digitales propiedades casi mágicas para mejorar la transparencia de los gobiernos, especialmente en el campo de la eficiencia divulgativa. Como con cualquier otra tecnología, existen una serie de factores que deben ser controlados para obtener resultados eficaces. El Premio Nobel de Economía Ronald Coase afirmó que los aspectos del sistema económico que son difíciles de medir tienden a descuidarse (Coase, 1994). Tan sensata afirmación debería extenderse a aquellos aspectos difíciles de comunicar, para

\section{REFERENCIAS}

Abella, A., Ortiz-de-Urbina-Criado, M., De-Pablos-Heredero, C. (2019). The process of open data publication and reuse. Journal of the Association for Information Science and Technology, 70(3), 296-300. https://doi. org/10.1002/asi.24116

Baack, S. (2015). Datafication and empowerment: How the open data movement re-articulates notions of democracy, participation, and journalism. Big Data \& Society, 2(2), 2053951715594634. https://doi. org/10.1177/2053951715594634

Benkler, Y. (2011). A Free Irresponsible Press: Wikileaks and the Battle Over the Soul of the Networked Fourth Estate. Harvard Civil Rights-Civil Liberties Law Review. https://dash.harvard.edu/handle/1/10900863 https://doi.org/10.7146/politik.v15i2.27508

Brandusescu, A., Iglesias, C., Lämmerhirt, D., Verhulst, S. (2019). Open data governance and open governance: Interplay or disconnect? World Wide Web Foundation. https://webfoundation.org/2019/02/ open-data-governance-and-open-governance-interplay-or-disconnect/

Cárcaba García, A., García García, J. (2003). La información financiera en Internet de las corporaciones locales europeas. Partida doble, 13(144), 56-69.

Charnock, G., March, H., Ribera-Fumaz, R. (2019). From smart to rebel city? Worlding, provincialising and the Barcelona Model. Urban Studies, OnlineFirst. https:// doi.org/10.1177/0042098019872119

Chen, Y. N., Chen, H. M., Huang, W., Ching, R. K. H. (2006). E-Government Strategies in Developed and Developing Countries: An Implementation Framework and Case Study. Journal of Global Information Management (JGIM), 1. https://doi.org/10.4018/ jgim. 2006010102

Clarke, A., Francoli, M. (2014). What's in a name? A comparison of 'open government' definitions across seven Open Government Partnership members. JeDEM - EJournal of EDemocracy and Open Government, 6(3), 248-266. https://doi.org/10.29379/ jedem.v6i3.227

Coase, R. H. (1994). Essays on economics and economists. Univ. of Chicago Press.

de la Cueva González-Cotera, J. (2019). La configuración del software como cuestión política. Teknokultura. Revista de Cultura Digital y Movimientos Sociales, 16(2), 159-180. https://doi.org/10.5209/TEKN.63795 impedir que la ciudadanía descuide sus legítimos derechos de evaluación y control de gobiernos y administraciones

\section{NOTAS}

1. Presupuestos Abiertos del Ayuntamiento de Madrid : https://presupuestosabiertos.madrid.es/es/

2. Datos Económicos de la Diputación de Castellón: https://datoseconomicos.dipcas.es/

3. Repositorio del proyecto en https://github.com/aragonopendata/presupuesto

4. Repositorio de adaptaciones de Fundación Ciudadana Civio en https://github.com/civio

Fuchs, C. (2010). Alternative Media as Critical Media. European Journal of Social Theory, 13(2), 173-192. https://doi.org/10.1177/1368431010362294

García-García, J. (2014). Gobierno abierto: Transparencia, participación y colaboración en las administraciones públicas. Innovar, 24(54), 75-88. https://doi. org/10/gcx5v5

García-García, J., Curto-Rodríguez, R. (2018). Divulgación de información pública de las comunidades autónomas españolas (2013-2017): Portal de datos abiertos, portal de transparencia y web institucional. El Profesional de la Información, 27(5), 1051. https:// doi.org/10.3145/epi.2018.sep.09

García-García, J., Curto-Rodríguez, R. (2019). El ejercicio de la rendición de cuentas mediante portales de datos abiertos en las comunidades autónomas españolas. IDP. Revista de Internet, Derecho y Política, 29. https:// www.raco.cat/index.php/IDP/article/view/373562

González-Limón, M., Rodríguez-Ramos, A. (2019). Revisión del cumplimiento de los Datos Abiertos por los Ayuntamientos españoles en la iniciativa Aporta. Revista Española de Documentación Científica, 42(4), e247. https://doi.org/10.3989/redc.2019.4.1605

Gray, J. (2015). Open Budget Data: Mapping the landscape. Open Knowledge. http://www.fiscaltransparency.net/ eng/resource_open_public.php?IdToOpen $=20150902128$ https://doi.org/10.2139/ssrn.2654878

Gutiérrez, M. (2018). Data activism and social change. Springer Berlin Heidelberg. https://doi.org/10.1007/9783-319-78319-2

Heald, D. (2003). Fiscal Transparency: Concepts, Measurement and UK Practice. Public Administration, 81(4), 723759. https://doi.org/10.1111/j.0033-3298.2003.00369.x

Heald, D. (2012). Why is transparency about public expenditure so elusive? International Review of Administrative Sciences, 78(1), 30-49. https://doi.org/10/gcx5x2

Heimstädt, M. (2017). Openwashing: A decoupling perspective on organizational transparency. Technological Forecasting and Social Change, 125, 77-86. https:// doi.org/10.1016/j.techfore.2017.03.037

Johnson, N. F., Velásquez, N., Restrepo, N. J., Leahy, R., Gabriel, N., Oud, S. E., Zheng, M., Manrique, P., Wuchty, S., Lupu, Y. (2020). The online competition between pro- and anti-vaccination views. Nature, 1-4. https:// doi.org/10.1038/s41586-020-2281-1 
Kahneman, D. (2012). Thinking, fast and slow. Penguin Books.

Kiess, H. O., Green, B. A. (2009). Statistical Concepts for the Behavioral Sciences (4th ed.). Pearson.

Lazer, D. M. J., Baum, M. A., Benkler, Y., Berinsky, A. J., Greenhill, K. M., Menczer, F., Metzger, M. J., Nyhan, B., Pennycook, G., Rothschild, D., Schudson, M., Sloman, S. A., Sunstein, C. R., Thorson, E. A., Watts, D. J., Zittrain, J. L. (2018). The science of fake news. Science, 359(6380), 1094-1096. https://doi.org/10.1126/ science.aao2998

Meijer, A. (2013). Understanding the Complex Dynamics of Transparency. Public Administration Review, 73(3), 429-439. https://doi.org/10/f4xbkv

Moon, M. J. (2002). The Evolution of E-Government among Municipalities: Rhetoric or Reality? Public Administration Review, 62(4), 424-433. https://doi. org/10/frssdj

Morozov, E. (2013). To save everything, click here: Technology, solutionism, and the urge to fix problems that don't exist. Penguin UK.

Nielsen, R. K., Graves, L. (2017). News you don't believe: Audience perspectives on fake news. https:// reutersinstitute.politics.ox.ac.uk/sites/default/ files/2017-10/Nielsen\&Graves_factsheet_1710v3_ FINAL_download.pdf

O'Donell, G. A. (1994). Delegative Democracy. Journal of Democracy, 5(1), 55-69. https://doi.org/10.1353/ jod. 1994.0010

Parks, W. (1957). Open Government Principle: Applying the Right to Know Under the Constitution. George Washington Law Review, 26(1), 1-77.

Rodríguez, D. (2013). Memecracia: Los virales que nos gobiernan. Gestión 2000.
Royo-Montañés, S., Benítez-Gómez, A. (2019). Portales de datos abiertos. Metodología de análisis y aplicación a municipios españoles. El Profesional de la Información, 28(6), e280609. https://doi.org/10.3145/ epi.2019.nov.09

Tarhini, A., Arachchilage, N. A. G., Masa'deh, R., Abbasi, M. S. (2015). A Critical Review of Theories and Models of Technology Adoption and Acceptance in Information System Research: International Journal of Technology Diffusion, 6(4), 58-77. https://doi.org/10.4018/ IJTD. 2015100104

Torres, L., Pina, V., Acerete, B. (2005). E-government developments on delivering public services among EU cities. Government Information Quarterly, 22(2), 217238. https://doi.org/10.1016/j.giq.2005.02.004

Vosoughi, S., Roy, D., Aral, S. (2018). The spread of true and false news online. Science, 359(6380), 11461151. https://doi.org/10.1126/science.aap9559

Wardle, C., Derakhshan, H. (2017). Information disorder: Toward an interdisciplinary framework for research and policy making (DGI(2017)09). Council of Europe. http://edoc.coe.int/en/media/7495-information-disorder-toward-an-interdisciplinary-framework-for-research-and-policy-making.html

Woolley, S. C., Howard, P. N. (2016). Political Communication, Computational Propaganda, and Autonomous Agents. International Journal of Communication, 10, $4882-4890$

Worthy, B. (2015). The Impact of Open Data in the UK: Complex, Unpredictable, and Political. Public Administration, 93(3), 788-805. https://doi.org/10/f7rr53

Young, R. K., Veldman, D. J. (1972). Introductory statistics for the behavioral sciences. Holt, Rinehart and Winston.

Yu, H., Robinson, D. G. (2012). The New Ambiguity of "Open Government". UCLA Law Review, 59, 178-208. https://doi.org/10.2139/ssrn.2012489. 